\title{
Research on Classroom Practice
}

\author{
Yeping Li and Hélia Oliveira
}

\section{Introduction}

Classroom practice, as a process, involves multiple agents and their interactions within the classroom as a system. The process can be manifested in diverse formats and structures, and its effectiveness can be influenced by numerous factors both internal and external to the classroom. Research on (mathematics) classroom practice can thus take different perspectives, and much remains to be examined and understood as we all try to improve mathematics teaching and learning through classroom practice.

Although it has long been recognized that research on classroom practice is important, large-scale systematic research on classroom practice in school mathematics is a relatively new endeavour. In fact, this Topic Study Group is only the second time in the ICME history to take a primary focus on classroom practice. As the quality of classroom instruction is a key to students' mathematics learning, this Topic Study Group focuses on finding ways for understanding, assessing, and improving the quality of classroom practice.

The entire organizing team worked together before the congress in planning and organizing TSG 21. The TSG 21 was well attended in all four 90-min sessions,

Organizers Team Chairs: Yeping Li (USA), Hélia Oliveira (Portugal); Team Members: Merrilyn Goos (Australia), Kwangho Lee (Korea), Raimundo Olfos (Chile); Liaison IPC Member: Fredrick Leung (Hong Kong).

\footnotetext{
Y. Li (ه)

Texas A\&M University, College Station, USA

e-mail: yepingli@tamu.edu

H. Oliveira

University of Lisbon, Lisbon, Portugal

e-mail: hmoliveira@ie.ul.pt

(C) The Author(s) 2015 
which indicates strong interest in this topic by congress delegates. This report provides an overview of the aim and focus of TSG21 and a summary of the discussion that occurred throughout the sessions.

\section{Aims, Focuses, and Themes}

As set by the organization team, the general aim of TSG 21 was, in the international mathematics education community, to elevate people's understanding of the importance, specific nature, and challenges in research on classroom practice, to promote exchanges and collaborations in identifying and examining high-quality practices in classroom instruction across different education systems, and to enhance the quality of research and classroom practice. More specifically, through its official program during the congress and other activities (including those before and after the congress), TSG 21 was intended to provide an international platform for all interested parties (e.g., mathematics educators, mathematics teachers, educational researchers, etc.) to disseminate findings from their research on classroom practice with the use of various theoretical perspectives and methodologies, and to exchange ideas about mathematics classroom research, development, and evaluation.

The main focus of TSG 21 was a discussion of research related to mathematics classroom practice, which includes activities of learning and teaching processes located within the classroom as a system. This requires a study of the interactions among the mathematical content to be taught and learned, the instructional practices of the teacher, and the work and experiences of the students. In the interaction processes, mathematical content is contextualized through situations, the teacher plays an important instructional role drawing on his/her knowledge, and the students involve themselves in the learning processes. It is important to understand through research the nature and extent of these interactions, the complexity of the didactic system, the roles of the teacher and students in the interaction processes when the mathematical content is taught and learned, and the complexity of the activities in the classroom.

The 39 accepted papers were assembled into the following eight themes for presentation and discussion during the congress:

- Theme 1: Theoretical and methodological considerations

- Theme 2: Instructional context, reflection, and improvement

- Theme 3: High-quality instructional practices

- Theme 4: Students' perception, class work, and learning

- Theme 5: Teaching and learning elementary mathematics

- Theme 6: Teachers' questioning and response in classroom instruction

- Theme 7: Instructional design and practice

- Theme 8: Curriculum/task implementation

In addition, there were nine proposals accepted for poster presentations in a separate session organized by the Congress. 
Each of the four 90-min sessions (July 10, 11, 13, and 14 in 2012) was devoted to two of these eight themes (4-5 papers for each theme), which were carried out simultaneously in two separate rooms. In the following sections, we briefly summarize the paper presentations and discussions during these sessions.

\section{Session 1 (Theme 1: Theoretical and Methodological Considerations)}

Gade adopted a theory/practice approach based on Vygostky for researching classroom practice, with the potential of informing practitioner's inquiry in ongoing classrooms. Morera and Fortuny illustrated the use of an analytical method of classroom episodes as a proposal to develop systematic research on whole-group discussions. Mesa, Lande and Whittemore argued for the need to attend to two dimensions of classroom interaction when describing it, by one study where they, simultaneously, analyzed the complexity of mathematical questions and the interactional moves that the teachers use to encourage student involvement in the lesson. Canavarro, Oliveira and Menezes illustrated the use of an analytical tool for lessons driven by an inquiry-based perspective in the case of one teacher who adopted a four phase model for the lesson structure. Xolo reported one expanded coding scheme that focuses on learning outcomes and teachers' didactic strategies from video recordings of sequences of lessons, intended to capture a greater degree of nuance in classroom practice.

In synthesis, these papers propose new analytical tools to investigate the classroom practice that contribute to having a better picture of what is happening in the classroom, showing a deep concern for acknowledging the teachers' work.

\section{Session 1 (Theme 2: Instructional Context, Reflection, and Improvement)}

Andersson presented a study of disengaged students' identity narratives in the senior secondary years. The instructional context was defined by tasks, situations (tools, activities, participants), school structures, the socio-political context, and the societal context. Olfos and Estrella described the use of a short video rich in potential problem situations to help primary school teachers initiate a lesson on fractions via problem posing. The lesson study approach resulted in the lesson being successively improved as each teacher implemented it. Oliveira, Menezes, and Canavarro reported on a project that created multimedia cases to stimulate reflective analysis of lessons in teacher education. Lee and Kim analysed one teacher's discourse during lessons involving small group work and used the lesson video to stimulate the teacher's reflective thinking towards improvement. Vanegas, 
Giménez, and Font i Moll illustrated the use of a two dimensional grid for identifying nine types of democratic mathematical practices in the classroom.

In synthesis, each of the papers presented in this theme reported on attempts to support democratic, equitable and critical classroom practices. The authors also investigated processes of teacher change by supporting teachers' systematic reflection and iterative improvement of their lessons.

\section{Session 2 (Theme 3: High-Quality Instructional Practices)}

Zhao and Ma found that lessons taught decades apart had similar content and teacher-student interaction but different types of tasks. Lee reported on the classroom practice of a teacher with high levels of mathematical knowledge for teaching. This study highlights a need for sensitivity in building respectful relationships between the researcher and teacher when classroom observation is also used for teacher evaluation. Zhao examined secondary school teaching practices in China where few teachers have a background in statistics. Focusing on teachers' interpretation of statistical graphs, the study found that teachers had limited understanding of key statistical concepts and gave more emphasis to procedures than conceptual understanding. Lewis, Corey, and Leong compared research from Japan, Singapore, and the US and found similarities in the categories used to define high quality practice. Li asked what could be learned from culturally valued classroom practices in China, and proposed a framework comprising macro pedagogy and micro pedagogy perspectives for understanding classroom instruction.

In synthesis, the papers in this theme proposed a variety of analytical frameworks for observing lessons and evaluating the quality of instruction. But each was concerned with the question of what counts as "high quality" instruction, and whether there are common or different criteria across countries and cultures.

\section{Session 2 (Theme 4: Students' Perception, Class Work, and Learning)}

Olteanu presented some results from a longitudinal study whose aim was to provide and develop a repertoire of reliable practices and tools to solve immediate problems in teachers' daily professional lives; namely, to improve students' learning in mathematics. Gao and Tian concluded that the students in the class where an open inquiry to problem solving was adopted were more accurate and succinct, quicker, and more fluent in language than the students in the class that followed a guided inquiry. Yang and Leung found that secondary students generally do not perceive their mathematics classroom environment very favorably. Gender differences were also found. Yau and Mok reported five consecutive lessons that showed that most 
students imitated the teacher's examples completely or partly. The authors argued that the strong direct role of teacher might help the students master their mathematical content. Araya, Varas, Giaconi and Foltz analyzed pupil's perceptions about mathematics, math learning and teaching in Chile and Finland. Considering the significant difference between these two countries, results showed surprising similarities connected to prototypical ideas.

\section{Session 3 (Theme 5: Teaching and Learning Elementary Mathematics)}

Silvestre and Ponte showed that the teaching/learning experience supports the conjecture that proportional reasoning develops when students explore, solve problems, and work with different representations. Yong, Zanzali, and Jiar showed that by developing a favorable learning environment and through scaffolding the students (low achievers) could progressively adapt themselves to a child-centered approach and begin to think more autonomously. Goos, Geiger, and Dole presented a model of numeracy whose elements comprise mathematical knowledge, dispositions, tools, contexts, and a critical orientation to the use of mathematics, and applied it to analyze changes in one teacher's planning, classroom practice, and personal conceptions of numeracy. Kwon and Thames showed that despite variations in the use of the task and the collective work with students, the work of teaching involves several core features: hearing mathematical reasoning, mathematical needs, and key mathematical concepts; and comparing different solutions and making alternative solutions reasonable. Pinto studied the development of the meaning of multiplication and division of non-negative rational numbers, arguing that problem solving helps students to overcome some difficulties and to understand and to formalize mathematical concepts.

In synthesis, these papers illustrate good practices that draw on the use of powerful mathematical tasks alongside with approaches that promote students' autonomy and critical orientation in solving problems.

\section{Session 3 (Theme 6: Teachers' Questioning and Response in Classroom Instruction)}

Lee analyzed the changes in one pre-service teacher's questioning practices, as she starts to give her students the opportunity for explaining and justifying their mathematical ideas. Subramanian illustrated several forms of questioning by one Indian teacher, which she argues is a culture-influenced pedagogy in that country and thereby widely practiced in the classrooms. Fox reported how two teachers who were observed throughout one unit of instruction were able to handle unanticipated questions by posing counter examples or simpler related questions. Sun compared 
the questioning practices of two teachers (Chinese and Czech) by observing the video of one lesson from each teacher. The questions posed by the Chinese teacher tended to require only a short answer, in a short period of time, and without the teacher's help. On the contrary, the questions by the Czech teacher were more cognitively demanding, but he provided no scaffolding. Aizikovitsh-Udi, Star, and Clarke presented two case studies demonstrating that good teacher questioning involves more than just good questions.

In synthesis, these papers show the growing interest in the teachers' questioning practices as a consequence of the recognition of its pedagogical value for the students' learning. Some professional cultures seem to value the power of questioning for a long time, but the nature and objectives of the questions the teachers pose differ substantially from setting to setting.

\section{Session 4 (Theme 7: Instructional Design and Practice)}

Mogensen shared recent efforts in Denmark to focus on mathematical pedagogical goals and mathematical points in mathematics teaching. Choquet analyzed the practice changes of a primary school teacher resulted from using 'problème souverts' (open problems). Sekiguchi examined how Japanese mathematics teachers handle multi-dimensions of coherence and coordinate coherence and variation. Japanese mathematics teachers seemed to achieve multidimensional coherence by utilizing a double-anchored process schema, and their deliberate use of variation seemed to facilitate students' reflection. Lin described a general procedure of conceptual variation via either diagram form (more on perceptual knowledge), or verbal/symbolic form (more on rational knowledge). A lesson plan of conceptual variation on the topic of elliptical definition was also given to illustrate how to use the general procedure to design conceptual variation. Varas, Martínez, Fuentealba, Näveri, Ahtee, and Pehkonen presented results from a three-year follow-up Finland-Chile research project that introduced open-ended problem solving activities in third grade classes.

In synthesis, these papers present different perspectives and approaches used in developing and designing classroom instruction, with particular focuses on the use and organization of mathematical ideas/points, open-ended problems, instructional coherence and variation.

\section{Session 4 (Theme 8: Curriculum/Task Implementation)}

Huang, Li and Yang reported one study with three primary teachers in the context of the implementation of a new mathematics curriculum, in which the notion of variable was taught. All teachers promoted students' use of numbers and letters to describe realistic problems and explain conclusions, but they provided few opportunities for students to experience the problem-solving process. Moreira and 
Campelos discussed the implications of the implementation of a new mathematics curriculum on teachers' practices, focusing on the balance between the collective and individual component of the practice. Grow-Maienza presented the results of one program that promoted the teachers' integration of principles abstracted from a Korean curriculum into the curriculum in use in one elementary school in the USA. Bingolbali and Bingolbali analyzed one teachers' practice concerning the implementation of one task in the classroom, arguing that a low fidelity to the task plan may be an expression of the teacher's flexibility to attend to students' needs.

In synthesis, these papers show that curriculum reforms are fruitful contexts to research the classroom practice, that may provide good opportunities to rethink the professional development of teachers, but that it is also necessary to understand how the intended innovations relate with the collectively and individually established teachers' practices.

\section{Closing Remarks}

Among the main points discussed across the four sessions we highlight the following ones:

- The search for what characterizes "high-quality" practices and the frameworks used to evaluate these practices taking into account the cultural and national diversity;

- The evolving classroom practices in many countries that reflect a move from the traditional instruction to innovative ways of teaching, and the demanding teacher's role associated with that transformation;

- The practices of questioning and inquiry-based approaches in different countries and their commonalities and differences;

- The teachers' practices concerning the work with mathematical tasks, namely their concern about the role played by the contexts, and the students' dispositions and perspectives concerning mathematics;

- The "Chinese paradox" and other countries" paradoxes concerning the relationship between students' achievement and classroom practice;

- The development of new analytical tools to do research on classroom practice.

Naturally, in such a broad topic as classroom practice, many questions remain to be addressed. The diversity of themes and focuses presented suggests many different perspectives that contributors took on what constitutes "classroom practice", which aspects of classroom practice are to be focused, and how "practice" is conceived using different analytical frameworks. The participants shared a strong interest in continuing the TSG's dynamics, and proposed the possibility of exploring joint projects in different countries and new publications focusing on some of the main themes discussed, and of gathering at other international conferences to do informal meetings to continue to do some work together. 
Open Access This chapter is distributed under the terms of the Creative Commons Attribution Noncommercial License, which permits any noncommercial use, distribution, and reproduction in any medium, provided the original author(s) and source are credited. 